\title{
Horizontal and Vertical Scaling of Cloud Geometry Inferred from CloudSat Data
}

\author{
A. Guillaume, B. H. Kahn, Q. Yue, E. J. Fetzer, S. Wong, G. J. Manipon, H. Hua, And B. D. Wilson \\ Jet Propulsion Laboratory, California Institute of Technology, Pasadena, California
}

(Manuscript received 10 April 2017, in final form 7 March 2018)

\begin{abstract}
A method is described to characterize the scale dependence of cloud chord length using cloud-type classification reported with the 94-GHz CloudSat radar. The cloud length along the CloudSat track is quantified using horizontal and vertical structures of cloud classification separately for each cloud type and for all clouds independent of cloud type. While the individual cloud types do not follow a clear power-law behavior as a function of horizontal or vertical scale, a robust power-law scaling of cloud chord length is observed when cloud type is not considered. The exponent of horizontal length is approximated by $\beta \approx 1.66 \pm 0.00$ across two orders of magnitude $(\sim 10-1000 \mathrm{~km})$. The exponent of vertical thickness is approximated by $\beta \approx 2.23 \pm 0.03$ in excess of one order of magnitude $(\sim 1-14 \mathrm{~km})$. These exponents are in agreement with previous studies using numerical models, satellites, dropsondes, and in situ aircraft observations. These differences in horizontal and vertical cloud scaling are consistent with scaling of temperature and horizontal wind in the horizontal dimension and with scaling of buoyancy flux in the vertical dimension. The observed scale dependence should serve as a guide to test and evaluate scale-cognizant climate and weather numerical prediction models.
\end{abstract}

\section{Introduction}

While clouds are generally considered to contribute an overall positive feedback to climate warming (IPCC 2013), there remains much disagreement among different climate models (e.g., Zelinka et al. 2016) about the magnitude and the contributions of different cloud types, and their underlying dynamical, thermodynamic, and microphysical states. Recent studies have suggested that low-latitude convective aggregation may increase in the future (e.g., Wing and Emanuel 2014; Mauritsen and Stevens 2015; Bony et al. 2015; Holloway et al. 2017), implying a systematic change in scale-dependent cloud statistics. However, Noda et al. (2014) show that the frequency of small-scale high clouds increases more rapidly than large-scale high clouds under a global warming scenario using the Nonhydrostatic Icosahedral Atmospheric Model (NICAM). These modeling studies suggest a need for quantitative assessments of scaledependent statistics of cloud geometry in present-day observations.

The scale dependence of atmospheric fields and their variability are routinely explored using observations, numerical simulations, and theoretical models (e.g.,

\footnotetext{
Corresponding author: Alexandre Guillaume, alexandre.guillaume@ jpl.nasa.gov
}

Lovejoy 1982; Nastrom and Gage 1985; Nastrom et al. 1986; Cahalan et al. 1994; Tjemkes and Visser 1994; Pierrehumbert 1996; Cho et al. 1999; Wood and Taylor 2001; Lindborg 2007; Kahn and Teixeira 2009; Bacmeister and Stephens 2011; Kahn et al. 2011; Pressel and Collins 2012; Fischer et al. 2013; Pressel et al. 2014; Barker et al. 2017). Wood and Field (2011) show that the probability distribution function (PDF) of horizontal cloud sizes from the Moderate Resolution Imaging Spectroradiometer (MODIS) follows a power law characterized by an exponent of approximately $-5 / 3$. Equally relevant is the scale dependence of clear scenes between the clouds, as these regions are essential for infrared cooling to space (Mauritsen and Stevens 2015). Kiemle et al. (2015) show with Cloud-Aerosol Lidar and Infrared Pathfinder Satellite Observations (CALIPSO) data that cloud and clear-sky horizontal scale-dependent distributions are essentially indistinguishable between 5 and $1000 \mathrm{~km}$.

Estimates of temporal and spatial dependence of variability among atmospheric parameters are used to develop and improve subgrid parameterizations in weather and climate global circulation models (GCMs; e.g., Cusack et al. 1999). Observations of scale dependence are also crucial for validating the fidelity of geophysical variability simulated by models at the smallest resolved scales (e.g., Skamarock 2004; Takahashi et al. 2006; Hamilton et al. 2008). Furthermore, observed scaling behavior is used to 
tailor stochastic models to capture a specific scale dependence. The bounded cascade model introduced by Cahalan et al. (1994) is able to generate the $k^{-5 / 3}$ power spectrum of liquid water or optical depth. The ability of stochastic models to reproduce a power-law behavior of particular atmospheric properties offers a practical approach to representing subgrid phenomena within GCMs. These same stochastic models are also useful for representing cloud-scale dependence in three-dimensional radiative transfer calculations at the subgrid scale (Davis and Marshak 2010). So, in addition to the cloud information they provide, exponents derived from scaling of observed cloud quantities can be used in stochastic models to generate cloud fields in a computationally efficient manner.

This work describes the scaling of CloudSat profile chord length by quantifying each CloudSat cloud-type distribution and, independently, cloud and clear-sky distributions. While numerous investigations have focused on the horizontal scaling of cloud imagery (e.g., Wood and Field 2011), or the horizontal scaling of thermodynamic profiles (e.g., Kahn and Teixeira 2009; Pressel and Collins 2012; Fischer et al. 2012), we attempt to separately derive the horizontal and vertical scaling exponents of cloud-chord-length PDFs. To date, very little attention has been paid to the scaling behavior in the vertical dimension, especially for clouds; some exceptions are noted for aerosols and wind shear. The atmospheric aerosol backscatter ratio measured from an airborne lidar platform (Lilley et al. 2004) was used to demonstrate the anisotropy of the backscatter scaling with a horizontal exponent $\beta_{h}=1.61 \pm 0.05$ and a vertical exponent $\beta_{v}=2.15 \pm 0.05$. In Lovejoy et al. (2007), dropsonde data was analyzed and the vertical wind shear, the difference of horizontal wind with altitude, was shown to have a vertical exponent $\beta_{v} \approx 2.4$. To our knowledge, a benchmark of the vertical scaling of cloud thickness does not currently exist. The need for one motivates this work, which builds on exponents obtained for cloud volume using Cloudsat data (Bacmeister and Stephens 2011). Global climate models struggle to simulate the vertical distributions of shallow- and deep-cloud hydrometeors, the thermodynamic environment, and the circulation in which clouds are embedded (Bony et al. 2015). Section 2 introduces the CloudSat data. Section 3 details the cloud horizontal and vertical length scale distributions for both individual cloud types and for all clouds regardless of type. Implications are discussed in section 4 and a summary is presented in section 5 .

\section{Data}

CloudSat was launched on 28 April 2006 to profile the vertical structure of clouds and light precipitation globally for the first time. The nadir-pointing 94-GHz Cloud Profiling Radar (CPR) observes profiles of backscattered power from clouds and precipitation hydrometeors (Stephens et al. 2002). The radar reflectivity $Z$ measured by the CPR is used to retrieve physical quantities such as vertical cloud structure, precipitation, radiative heating and flux profiles, and cloud water content (CWC). The algorithms are simplified by identifying different classes of physical conditions (cloud classes or types), then selecting the appropriate retrieval algorithm flow. To this end, Sassen and Wang (2005) devised an algorithm to classify clouds into eight cloud classes that include cumulus $(\mathrm{Cu})$, stratocumulus $(\mathrm{Sc})$, stratus $(\mathrm{St})$, altocumulus (Ac), altostratus (As), nimbostratus (Ns), cirrus (Ci), and deep convective (DC) clouds. Although we consider the St cloud type in the remainder of this work as other cloud types, it is worth noting that CloudSat underestimates these low-level clouds because of CPR signal contamination in the lowest three or four vertical bins $(0.75-1.0 \mathrm{~km})$ above the surface (Sassen and Wang 2008). The two-dimensional CloudSat field, cloud_ scenario, used to calculate the scaling results is contained within the 2B-CLDCLASS file (http://cloudsat. atmos.colostate.edu/). Each CPR profile is horizontally separated by $1.1 \mathrm{~km}$ in the along-track direction. There are 125 bins in each vertical profile, with each bin approximately $240-m$ thick. In the next section, we describe how the horizontal and vertical cloud chord lengths are derived. Wood and Field (2011) observed that the cloud-chord-length distributions derived from the MODIS imager are essentially indistinguishable between the across-track and along-track directions, implying that the cloud-chord-length distributions are horizontally isotropic. Pressel and Collins (2012) found that temperature and specific humidity soundings from the Atmospheric Infrared Sounder (AIRS) instrument largely exhibit horizontal isotropy. Therefore, the twodimensional curtain sampling of CloudSat can be viewed as representative of the three-dimensional atmosphere. We show results for a 2-yr period starting on 1 July 2006 and ending on 30 June 2008.

\section{Methodology and results}

\section{a. Horizontal cloud-length distributions by cloud type}

The length of cloud systems in the horizontal is defined as the number of horizontally contiguous CloudSat profiles containing a given cloud type at any altitude in the profile, multiplied by the profile spacing of $1.1 \mathrm{~km}$. The process is illustrated in Fig. 1, where CloudSat cloud observations (Fig. 1a) are represented as rectangles (Fig. 1b) with widths being equal to the cloud-length defined above. The length distributions of each cloud 


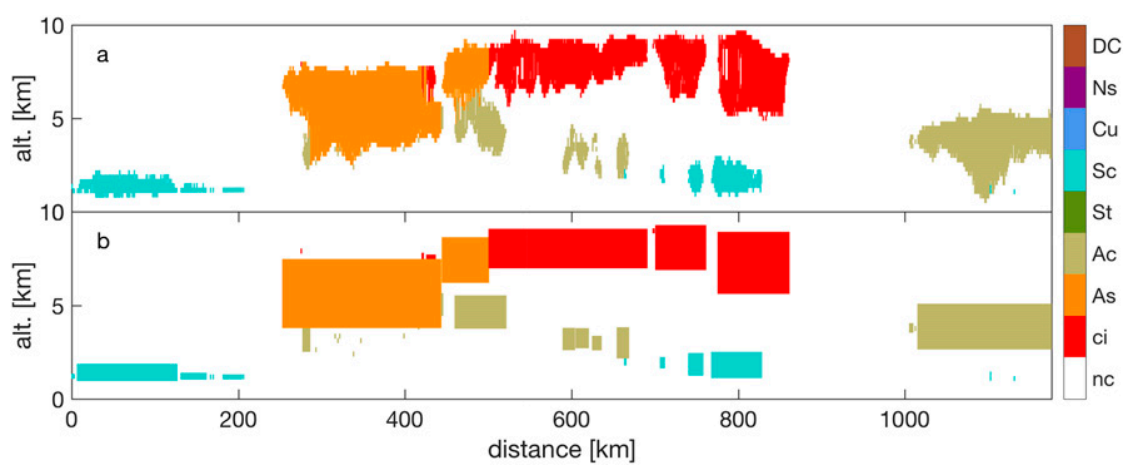

FIG. 1. (a) Cloud profile defined by the values of the cloud_scenario variable in 2B-CLDCLASS. (b) Fictitious rectangular clouds with dimensions equal to the length and average thickness of the corresponding clouds in (a). The cloud horizontal length was calculated by counting the number of horizontally contiguous CloudSat profiles with at least one bin of a given cloud type in the profile.

type are shown as frequency histograms in Fig. 2. A common feature of the length distributions of all cloud types except Ns and DC is that the smallest clouds are most numerous. This characteristic has been previously reported using satellite and in situ data (e.g., Wood and Field 2011). The mean, median, standard deviation, and mean absolute deviations of cloud horizontal length for each cloud type are summarized in Table 1.

\section{b. Horizontal cloud-length distribution regardless of cloud type}

Alternatively, cloud lengths are calculated regardless of cloud type. If a cloud is defined as a set of cloudy pixels between two clear columns, then the horizontal length of a cloud is simply the number of horizontally contiguous cloudy pixels between the clear boundaries. The gray and black segments at the top of Fig. 3 represent the succession of clear and cloudy scene lengths, respectively, corresponding to the clouds in the lower part of the figure. A tally of cloudy and clear scene lengths encountered within the 2-yr-long data record considered here yields the density histograms shown in
Fig. 4a; each data point is normalized by the bin width and the total number of points. The distribution of cloud horizontal lengths follows a clear power-law behavior $n(L) \sim L^{-\beta}$ (a straight line on a log-log plot) across two orders of magnitude from 10 to $1000 \mathrm{~km}$. For scales larger than approximately $1000 \mathrm{~km}$, the distribution departs from a power law.

A very similar behavior was observed by Wood and Field (2011) with cloud chord length evaluated using MODIS and aircraft data. The scale at which the cloud distribution deviates from power-law scaling, the scale break $L *$, is derived following Wood and Field (2011):

$$
n(L) \sim L^{-\beta} \exp \left[-\left(L / L_{*}\right)^{2}\right]
$$

The fit of the distribution of cloud length $n(L)$ with Eq. (1) is shown in Fig. 4b with a gray dashed line. Scales smaller than $10 \mathrm{~km}$ were excluded from the fit and are represented with black diamonds in Fig. 4b. The value of exponent $\beta$ deduced from a least squares fit is $1.66 \pm$ 0.00 and the scale break length $L *$ is approximately $1850 \mathrm{~km}$. Although the error here comes from the $95 \%$

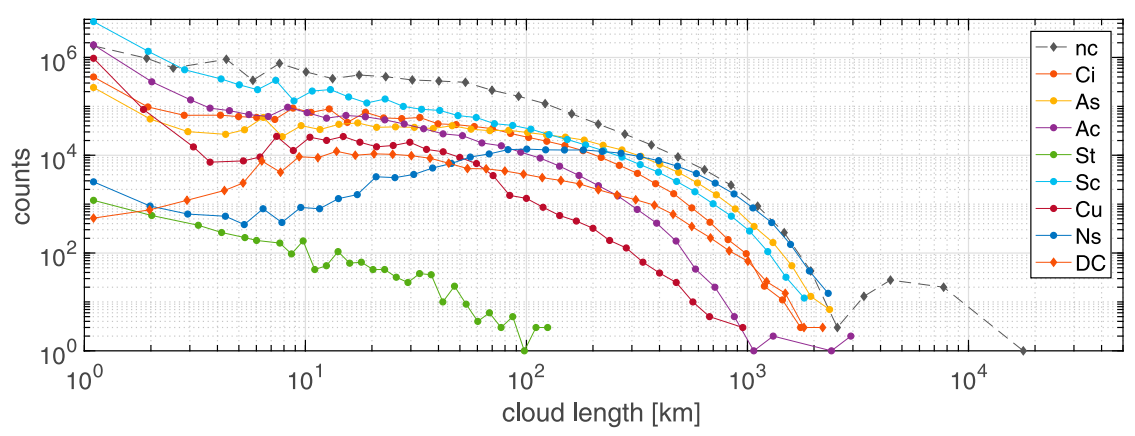

FIG. 2. Different cloud-type horizontal-length distributions. The horizontal length corresponds to the horizontal length of a fictitious cloud in Fig. 1b. 
TABLE 1. Mean horizontal length, standard deviation, median, and MAD for each cloud type. All values are in kilometers.

\begin{tabular}{lrrrrrrrrr}
\hline \hline & $\mathrm{NC}$ & $\mathrm{Ci}$ & $\mathrm{As}$ & \multicolumn{1}{c}{$\mathrm{Ac}$} & \multicolumn{1}{c}{$\mathrm{St}$} & $\mathrm{Sc}$ & $\mathrm{Cu}$ & $\mathrm{Ns}$ & $\mathrm{DC}$ \\
\hline Mean & 22.8 & 26.6 & 50.1 & 7.8 & 7.1 & 8.6 & 5.7 & 204.3 & 55.6 \\
Std dev & 61.0 & 54.0 & 98.1 & 21.4 & 11.2 & 31.1 & 14.8 & 210.7 & 92.8 \\
Median & 6.6 & 8.8 & 13.2 & 1.1 & 3.3 & 1.1 & 1.1 & 136.4 & 25.3 \\
MAD & 25.7 & 29.1 & 57.2 & 9.4 & 6.5 & 11.0 & 7.3 & 147.9 & 51.5 \\
\hline
\end{tabular}

confidence interval values provided by the fitting procedure, the value of the exponent itself is strongly dependent on the value of $L *$, which is highly undetermined. The scatter of the data points with respect to the power law is greatest at lengths smaller than $10 \mathrm{~km}$. Fits of power-law distributions can be difficult because they depend on the bin choices such as width or locations (Zhao and Di Girolamo 2007), so they can introduce systematic biases into the value of the exponent (Goldstein et al. 2004). Newman (2005) derived a simple analytic method to evaluate the exponent $\beta$ of a distribution $p(x)=C x^{-\beta}$ with a maximum likelihood estimate

$$
\beta=1+n \times\left[\sum_{i} \ln \left(x_{i} / x_{\min }\right)\right]^{-1} .
$$

The quantities $x_{i}, i=1, \ldots, n$ are the measured values of $x$, and $x_{\min }$ is the minimum value of $x$ (for which the power law holds). The uncertainty on the estimate given by Eq. (2) is, for large n (Clauset et al. 2009),

$$
\sigma \approx(\beta-1) / \sqrt{n}
$$

It is worth noting that the expression $\beta$ does not depend on bin properties such as the bin heights or locations. Thus, the 10-1000-km length range exponent using Eq. (2) is $\beta=1.74 \pm 0.00$, which is slightly larger than obtained from the fit using Eq. (1).

\section{c. Vertical cloud thickness distributions by cloud type}

The vertical cloud thickness was obtained for each cloud by averaging the thickness over all the vertical profiles within the cloud defined by the horizontal length (section 3a). The vertical thickness of a single profile is the number of contiguous vertical bins of the same cloud type times the bin thickness of $240 \mathrm{~m}$. This method is illustrated in Fig. 1b with rectangular clouds where vertical edge length is equal to the average vertical thickness, and horizontal edge length is equal to the horizontal length defined in section $3 \mathrm{a}$. The frequency histogram of thickness for each cloud type is displayed in Fig. 5 and summary statistics are presented in Table 2.

\section{d. Vertical cloud thickness distribution regardless of cloud type}

The distribution of vertical cloud thickness is derived regardless of cloud type (Fig. 6). The cloud thickness was calculated as described in section $3 c$ but the total count of thicknesses was performed over all cloud types. As with cloud lengths, the thickness distribution is highly skewed toward low values. A power-law exponent $\beta \approx 2.23 \pm 0.03$ was obtained using the least squares fit of Eq. (1) between 1.1 and $14 \mathrm{~km}$, but, as discussed previously, this procedure can lead to unreliable results. Therefore, the maximum likelihood estimate (Newman 2005) of Eq. (2) is also calculated leading to $\beta \simeq 2.38 \pm 0.00$. This value is different from 1.66 derived from horizontal scales, showing a distinct and robust anisotropy between vertical and horizontal cloud-chord-length scalings.

\section{e. Cloud distributions in the tropics and extratropics}

Previous investigations have suggested differences in scaling exponents between the tropics and extratropics (e.g., Nastrom and Gage 1985; Kahn and Teixeira 2009;

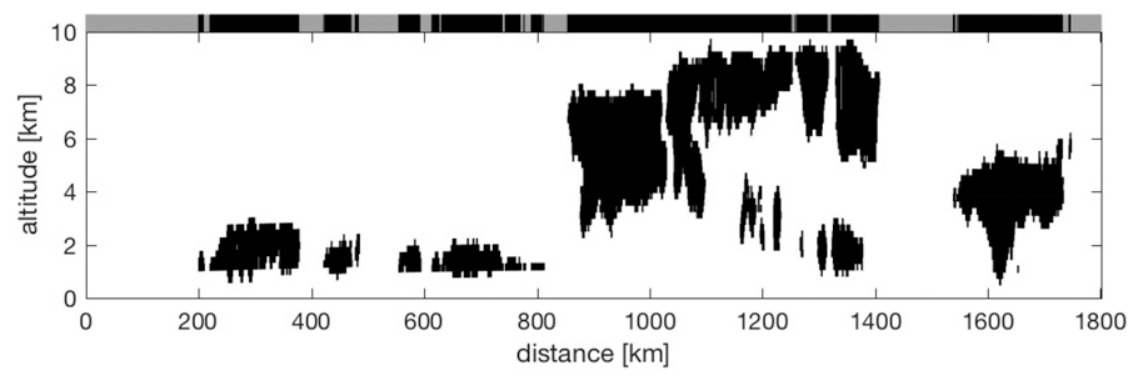

FIG. 3. Cloud profile defined by the values of the cloud_scenario variable in 2B-CLDCLASS. The clear type (NC) is white whereas all other eight cloud types are black. The length of a gray segment above the main panel is the horizontal length of the corresponding clear scene in the main panel and is equal to the number of CloudSat profiles that are totally clear (from the surface to the top). The horizontal length of a cloud is equal to the length of a black segment above the main panel. 

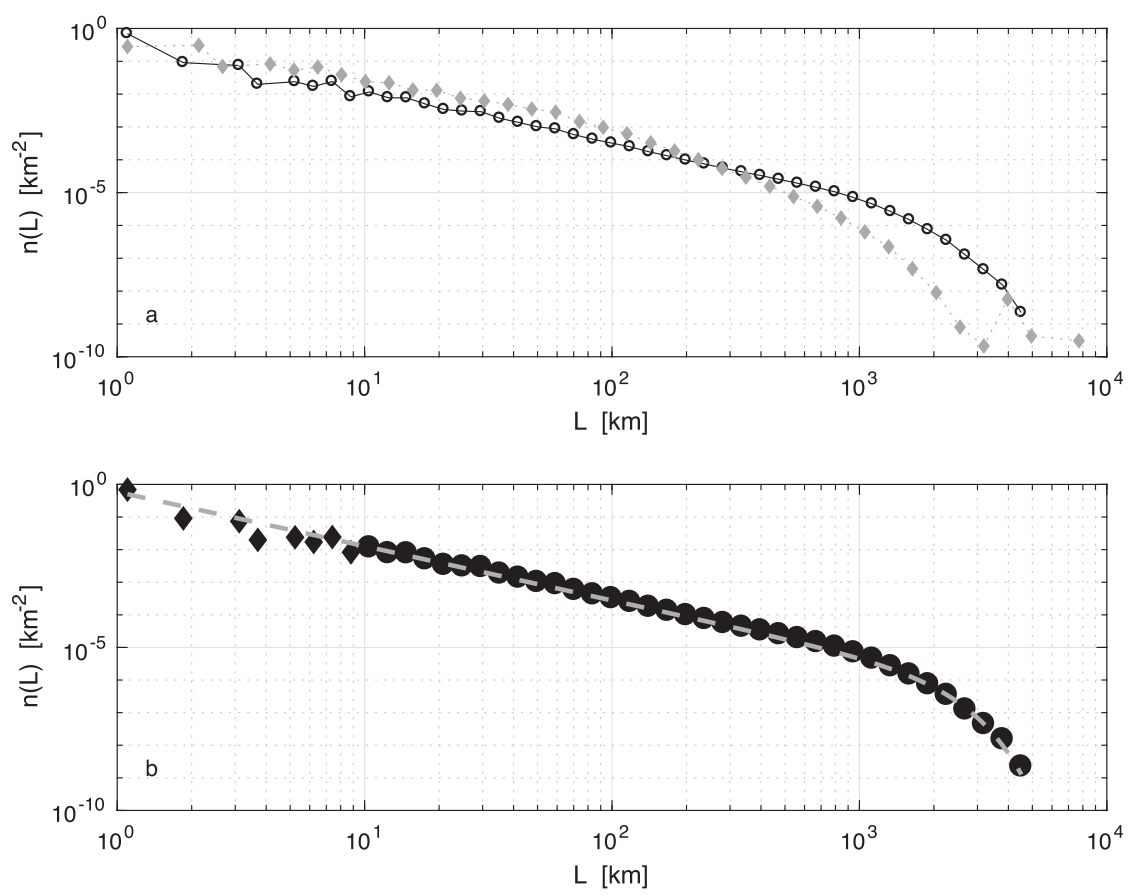

FIG. 4. (a) Cloud and clear-scene horizontal-length distribution $n(L)$ indicated with open circles and gray diamonds, respectively. The cloud (clear scene)-length distribution was obtained by counting the black (gray) segments on the top of Fig. 3. (b) The gray dashed line corresponds to a fit with $n(L) \sim L^{-\beta} \times \exp \left[-\left(L / L_{*}\right)^{2}\right]$. The exponent $\beta$ of the power is equal to $1.66 \pm 0.00$ and the scale break $L *$ is approximately equal to $1850 \mathrm{~km}$. The data points represented with black diamonds in (b) were excluded from the fit.

Fischer et al. 2013) for temperature and water vapor. To determine if there are differences in cloud-scaling properties for different latitude bands compared to the global scale, the analysis is repeated in three different geographical zones: the tropics $\left(15^{\circ} \mathrm{S}-15^{\circ} \mathrm{N}\right)$, the $\mathrm{SH}$ extratropics $\left(30^{\circ}-60^{\circ} \mathrm{S}\right)$, and the $\mathrm{NH}$ extratropics $\left(30^{\circ}-60^{\circ} \mathrm{N}\right)$. These particular latitude bands are assumed to best represent dominance by tropical deep convection and extratropical baroclinicity, respectively.

The horizontal length distributions for each cloud type are derived for each latitude band as in Fig. 2 but not shown here. There are a greater number of smallscale $(4 \lesssim L \lesssim 40 \mathrm{~km})$ Cu clouds in the tropics than in the extratropics. Concomitantly, there are a smaller number of long $(20 \lesssim L \lesssim 1000 \mathrm{~km})$ Ns clouds in the tropics than in the extratropics. Consequently, one would expect a steeper slope characterizing the horizontal cloud-length distribution in the tropics than in the extratropics. We repeated the analysis for Fig. 4 in the three different latitude bands (not shown) and obtained the following exponents for the SH extratropics, tropics, and $\mathrm{NH}$ extratropics, respectively: $\beta \simeq 1.57 \pm 0.00$, $\beta \simeq 1.67 \pm 0.00$, and $\beta \simeq 1.56 \pm 0.00$ from the least squares fitting of Eq. (1), and $\beta \simeq 1.76 \pm 0.00, \beta \simeq 1.81 \pm 0.00$, and $\beta \simeq 1.71 \pm 0.00$ from the maximum likelihood estimate of Eq. (2). Therefore, a slightly larger horizontal exponent $\beta$ in the tropics is confirmed from Eqs. (1) and (2).

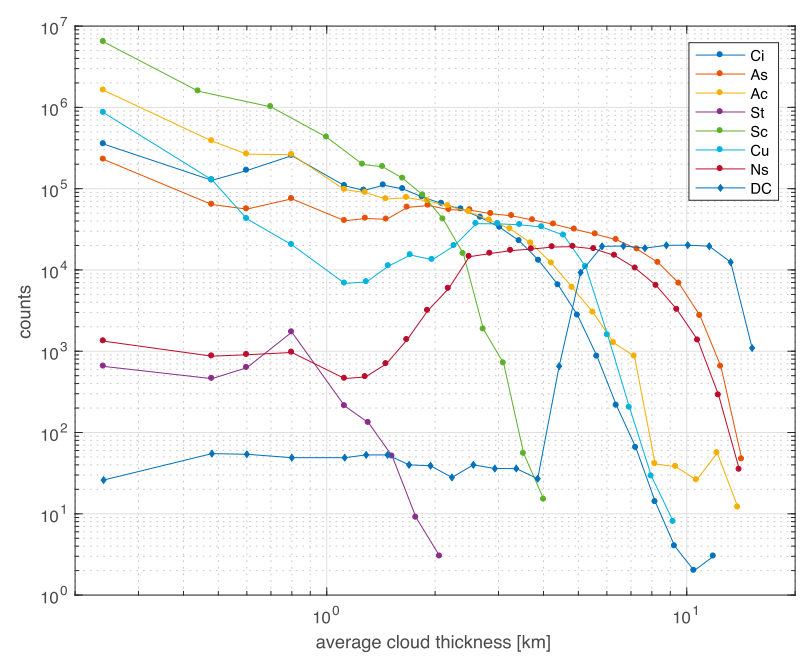

FIG. 5. Cloud thickness is calculated by averaging the vertical length of a given cloud type over all profiles included in a cloud (defined by adjacent CloudSat profiles with a given cloud type). This vertical length corresponds to the vertical length of a fictitious cloud in Fig. 1b. 
TABLE 2. Mean cloud thickness, standard deviation, median, and MAD for each cloud type. All values are in kilometers.

\begin{tabular}{lcccccccc}
\hline \hline & $\mathrm{Ci}$ & As & Ac & St & Sc & Cu & Ns & DC \\
\hline Mean & 1.2 & 2.2 & 0.8 & 0.8 & 0.5 & 0.9 & 4.7 & 9.3 \\
Std dev & 0.9 & 2.1 & 0.8 & 0.3 & 0.4 & 1.3 & 2.1 & 2.7 \\
Median & 1.0 & 1.6 & 0.5 & 1.0 & 0.2 & 0.2 & 4.5 & 8.9 \\
MAD & 0.7 & 1.6 & 0.6 & 0.3 & 0.3 & 0.9 & 1.7 & 2.3 \\
\hline
\end{tabular}

The vertical thickness distributions for each cloud type are derived for each latitude band (not shown) as in Fig. 5 . There are several interesting changes specific to some cloud types. Larger frequencies of occurrence of DC, Ac, $\mathrm{As}, \mathrm{Sc}$, and $\mathrm{Cu}$ clouds are found at nearly all length scales in the tropics compared to the extratropics. Given that DC is the least-abundant cloud type and that Ns is the second-least-abundant cloud type, the relative increase of $\mathrm{As}, \mathrm{Ac}, \mathrm{Sc}$, and $\mathrm{Cu}$ is more pronounced (on a log-log plot) at vertical lengths approximately greater than $2 \mathrm{~km}$ in the tropics compared to the extratropics. This results in a shallower slope of the vertical thickness distribution in the tropics compared to the extratropics. We repeated the analysis for Fig. 6 and obtained the following exponents for the $\mathrm{SH}$ extratropics, tropics, and $\mathrm{NH}$ extratropics, respectively: $\beta \simeq 2.45 \pm 0.04, \beta \simeq 1.92 \pm 0.04$, and $\beta \simeq 2.18 \pm 0.04$ from the least squares fitting of Eq. (1), and $\beta \simeq 2.36 \pm 0.00, \beta \simeq 2.09 \pm 0.00$, and $\beta \simeq 2.43 \pm 0.00$ from the maximum likelihood estimate of Eq. (2). Therefore, a significantly reduced vertical exponent $\beta$ in the tropics is confirmed from Eqs. (1) and (2).

\section{Discussion}

\section{a. Horizontal cloud-length distributions by cloud type and previous literature}

We first compare the horizontal cloud-length distributions by cloud type (section 3 a) with results published in the literature. Zhang et al. (2014) quantified the along-track spatial scales of Ac observed with collocated CALIPSO and CloudSat measurements. The present study differs in that combined CALIOP-CloudSat observations are used by Zhang et al. (2014) to identify Ac whereas the CloudSat-only Ac is used here. Furthermore, they exclude midlevel cloud systems with fewer than 10 profiles, whereas cloud types considered here are as small as $1.1 \mathrm{~km}$. The Ac mean length is $7.8 \mathrm{~km}$ with a standard deviation of $21.4 \mathrm{~km}$ (Table 1 ) using our approach. Zhang et al. (2014) find that the globalaverage Ac along-track horizontal scale is $40.2 \mathrm{~km}$ with a standard deviation of $52.3 \mathrm{~km}$. The difference essentially stems from clouds of horizontal extent smaller than 10 CloudSat profiles $(11 \mathrm{~km})$ and also from higher sensitivity to optically and geometrically thinner cloud features detectable by the CALIOP lidar. If clouds smaller than $11 \mathrm{~km}$ are neglected in this study, the mean Ac length is $55.6 \mathrm{~km}$ with a standard deviation of $43.7 \mathrm{~km}$, which compares well with Zhang et al. (2014). For lengths greater than $11 \mathrm{~km}$, the distribution of Ac lengths (purple curve in top of Fig. 2) is very similar to Fig. 2 in Zhang et al. (2014). The distribution is nearly flat or changes slowly for lengths between 10 and $100 \mathrm{~km}$, while it decreases faster for greater lengths until it reaches the longest observed length of slightly less than $1000 \mathrm{~km}$.

Kiemle et al. (2015) used CALIPSO data to evaluate the global distribution of cloud gaps. They set a cutoff cloud optical depth of 1 to discriminate between "cloud free" and "lidar cloudy" 5-km-resolution profiles. The length was calculated by tracking the number of sequential cloudy (or cloud-free) columns. The overall distribution of cloud-free lengths in Kiemle et al. (2015) is very similar to the no-cloud (NC) results found in this study (Fig. 2, gray curve). The vast majority of cloud-free gaps have small horizontal lengths. The frequency of gaps

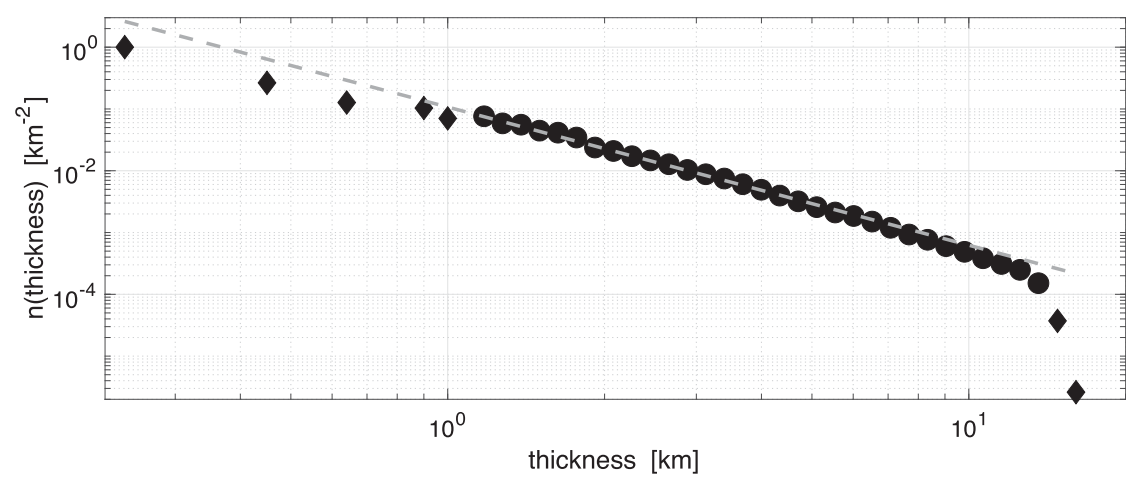

FIG. 6. Cloud-thickness distribution. The cloud vertical thickness corresponds to the vertical length of a (fictitious) rectangular cloud in Fig. 1b. The fit of the data (excluding the points represented by black diamonds) to a power law is shown with a gray dashed line. 
decreases with length down to a few rare occurrences approximately 3000-6000-km long. For lengths between about 2.2 and $300 \mathrm{~km}$, there are more cloud-free gap occurrences than clouds. For other lengths, there are more clouds than gaps. The median length of cloud-free gaps in Kiemle et al. (2015) is $10 \mathrm{~km}$ whereas the median length of NC indicated in Table 1 is $6.6 \mathrm{~km}$.

A surprising feature in Fig. 2 is the presence of DC systems with lengths in excess of $1000 \mathrm{~km}$, while Sassen and Wang (2005) indicate that a typical length between 10 and $50 \mathrm{~km}$ could be used as a tentative rule to classify a deep convective system. To shed some light on these large DC features, their locations were mapped. The locations of 84 deep convective clouds longer than $1100 \mathrm{~km}$ coincide with baroclinic systems (not shown) within two latitude bands of approximately $30^{\circ}-60^{\circ} \mathrm{S}$ and $30^{\circ}-60^{\circ} \mathrm{N}$ over oceans. The decision tree for precipitating cloud classification in Fig. 14 of Sassen and Wang (2005) does not appear to preclude clouds with lengths greater than $1000 \mathrm{~km}$ to be classified as DC. Sassen and Wang (2005) mention further decisions taken to separate DC from Ns. We point out that cloud classification is challenging, and predetermined algorithmic decision trees may reveal unexpected structure in observations.

\section{b. Horizontal cloud-length distributions regardless of cloud type and previous literature}

Next, we discuss the horizontal cloud-length distribution regardless of cloud type (section $3 \mathrm{~b}$ ) and compare it to the results of Wood and Field (2011), who employed a similar methodology with MODIS observations. The general shape of their distribution $n(L)$ follows our Eq. (1). A power-law behavior is obtained over three orders of magnitude (from 1 to $>2000 \mathrm{~km}$ ) with an exponent $\beta=1.66 \pm 0.04$ and a scale break $L *=$ $2100 \mathrm{~km}$. Given the vastly different sampling characteristics and instrument sensitivities, namely, passive MODIS reflectance over a wide range of viewing angles versus the vertically pointing active $94-\mathrm{GHz}$ CPR profiles, the results in Wood and Field (2011) and those presented herein are in remarkably good agreement.

However, there remains an important difference with Wood and Field (2011). None of the individual CloudSat cloud types have length distributions that follow apparent power-law behavior (i.e., Fig. 2). This result is in contrast with Wood and Field (2011), who used aircraft measurements of cloud liquid water content to derive cloud-length distributions of $\mathrm{Ci}, \mathrm{Sc}$, and $\mathrm{Cu}$, and showed that all three types exhibit power-law behavior with $\beta \approx 1.66$. One possible reason for this discrepancy is that the threshold nature of CloudSat cloud-type classification imposes unnatural boundaries between cloud types that may mask a robust power-law scaling behavior. Further research into cloud-type scaling behavior, or the lack of it, is warranted.

\section{c. Vertical cloud-length distributions and previous literature}

Next, we discuss the vertical cloud-length distributions by cloud type (section 3c) and compare them to Miller et al. (2014), who derived cloud-type vertical thickness from CloudSat. In their study, geometric thickness was determined as the difference between top and base heights for a vertically contiguous cloud layer within each CloudSat profile separately, using data spanning 2006-11. Miller et al. (2014) show statistics for each cloud type, seven latitude bands, and four seasons. While a more direct comparison of Miller et al. (2014) requires a weighting with respect to cloud-type occurrence frequency with latitude (Sassen and Wang 2008), only the mean vertical thickness of DC and Ns types are consistent between this work and Miller et al. (2014). Otherwise, the mean cloud thicknesses considered here are smaller for $\mathrm{Ci}, \mathrm{As}, \mathrm{Ac}, \mathrm{Sc}$, and $\mathrm{Cu}$ types. The standard deviations in Miller et al. (2014) and Table 2 are consistent, however. While the cause of the discrepancy in the mean cloud thickness is unclear, the differences may arise from the fundamentally different approaches taken in the calculations. All of the cloud types except Ns and DC have heavy-tailed distributions. This suggests that means and standard deviations are insufficient to characterize the distributions. A power-law distribution with $\beta$ between 2 and 3 does not have a meaningful variance according to Newman [2005; cf. their Eqs. (12) and (13)]. Thus, a direct comparison between Miller et al. (2014) and Table 2 is elusive. This motivates the inclusion of median and median absolute deviations (MADs) in Table 2.

We are not aware of any previous studies of vertical cloud-chord-length distribution regardless of cloud type (section 3d) and the exponent derived from it.

\section{d. Physical interpretation of derived exponents}

The $-5 / 3$ horizontal exponent has been observed for a wide variety of atmospheric quantities (as discussed in the introduction). Many studies have found significant deviations from the $-5 / 3$ scaling, however. Kahn and Teixeira (2009) and Pressel and Collins (2012) used temperature and specific humidity observations from the AIRS instrument on Aqua to derive scaling exponents, and showed that exponents strongly vary with latitude and altitude. Other studies have shown a horizontal exponent of -2 in specific humidity using an airborne differential absorption lidar (DIAL; Fischer et al. 2012), and in total water mixing ratio from numerical models over scales ranging from the synoptic scale $(\sim 1000 \mathrm{~km})$ to turbulent 
eddies in direct numerical simulation $(<1 \mathrm{~m}$; Schemann et al. 2013). Theoretically, the $-5 / 3$-exponent value is predicted by the Kolmogorov-Obukhov law that describes how energy in a turbulent flow passes from larger to smaller scales where viscosity is important and kinetic energy is dissipated (Landau and Lifshitz 1987, paragraph 33). The Kolmogorov-Obukhov law assumes homogeneous and isotropic turbulence. Although the Kolmogorov-Obukhov theory predicts that the power spectrum of a turbulent flow will follow a $k^{-5 / 3}$ law, we do not know any other simple physical model that would predict that the cloud size distribution should also follow a power law. There are, however, two types of model simulations that do: stochastic models and climate and weather numerical prediction models. The bounded cascade model of Cahalan et al. (1994) is a stochastic model whose parameters are chosen so that the resulting liquid water and optical depth spectra have a $-5 / 3$ power-law dependence. Wood and Field (2011) used the bounded cascade model to derive cloud size distributions with various exponents. Siebesma and Jonker (2000) used large-eddy simulations to derive geometric properties of cumulus clouds and found that those properties followed scaling laws. Additionally, O'Brien et al. (2013) showed that the Community Atmosphere Model (CAM) and the Weather Research and Forecasting Model (WRF) also exhibited horizontal cloud-scaling power-law exponents close to $-5 / 3$ at scales $>40 \mathrm{~km}$.

The uniqueness of the vertical cloud-chord-length exponent and its relation to the horizontal exponent is now described. The values of $\beta$ found in section $3 \mathrm{~d}$ are close to $-11 / 5$. The Bolgiano-Obukhov (BO) theory predicts that the potential temperature variance flux leads to a $k^{-11 / 5}$ spectrum. However, according to Lovejoy and Schertzer (2013), the isotropic BolgianoObukhov turbulence applies for scales greater than the characteristic scale $L_{\mathrm{BO}} \approx 10 \mathrm{~cm}$ and concomitantly, the isotropic 3D Kolmogorov law applies to scales smaller than $L_{\mathrm{BO}}$. Schertzer and Lovejoy $(1983,1985)$ proposed an alternative approach called the 23/9 dimensional Kolmogorov-Bolgiano-Obukhov theory that assumes the energy and buoyancy force fluxes coexist and cascade over a single wide-ranging scaling regime with the energy flux dominating in the horizontal and the buoyancy force flux dominating in the vertical.

There is empirical evidence supporting a vertical exponent of $-11 / 5$ and an anisotropy $\beta_{h} / \beta_{v}=(-5 / 3) /(-11 / 5)$. Anisotropy between the vertical and horizontal scaling exponents was obtained from aircraft measurements of the horizontal wind speed (Lovejoy et al. 2010). Power spectra and structure functions of the wind were used to derive $\beta=-5 / 3$ for scales smaller than $40 \mathrm{~km}$ and $\beta=-2.4$ for larger scales. Lovejoy et al. (2010) argued that the large-scale horizontal exponent value was consistent with the vertical scaling of the atmosphere using dropsondes. However, the horizontal scale break around $40 \mathrm{~km}$ observed in aircraft wind data was not observed in the temperature and humidity observations. They identified an optimum range of scales from 4 to $40 \mathrm{~km}$ where intermittency in the aircraft data was low enough to reliably estimate $\beta$. The estimate of $\beta$ obtained here is valid for two orders of magnitude between 10 and $1000 \mathrm{~km}$ using the fitting approaches in both Eqs. (1) and (2). The findings described in this work corroborate previous suggestions of 3D anisotropy in atmospheric scaling (Lovejoy et al. 2010). As clouds are controlled in large part by multiscale turbulent processes, the two distinct scaling exponents for the horizontal and vertical dimensions suggest that an anisotropic description of atmospheric turbulence is necessary.

Additionally, our results are consistent with Tuck (2010) when considering altitudes above 1-km altitude (the approximate vertical sensitivity range of CloudSat). The value of $\beta=2.38$ presented here corresponds to $H=0.69$, which is the value of the Hurst exponent $H$ cited in Tuck (2010; cf. their Fig. 3). The term $H$ is related to $\beta$ by $\beta=2 H+1$ when it describes a process that is scale-invariant and nonstationary. The results presented here and in Tuck (2010) have slightly larger values than predicted from variance buoyancy flux theory of $\beta=2.2(-11 / 5)$ but are consistent with each other. At levels $<1 \mathrm{~km}$, Tuck (2010) report $\beta=2.2(-11 / 5)$.

\section{e. Sampling and statistical caveats}

The exponents reported here are not exactly representative of the sampling found in aircraft data, which may fly inside, outside, and around clouds and may avoid convective clouds such as DC. CloudSat data provide continuous curtains of 2D cloud geometry along a constant-height grid. Outside of the tropics, the height grids increasingly deviate from isobars and isentropes. For convective processes that are fundamentally driven by buoyant parcels of air, it is reasonable to expect that buoyancy variance will approximately scale with cloud thickness. Within baroclinic systems, one might expect the scaling to differ from convection (e.g., Fischer et al. 2012), as the atmospheric flow is overwhelmingly horizontal; however, the anisotropic cloud scaling holds globally in this investigation. Horizontal structure functions of water vapor from lidar observations in convective and nonconvective regions (Fischer et al. 2013) were shown to have Hurst exponents $H=0.35 \pm 0.11$ and $H=0.63 \pm 0.10$, corresponding to the exponents $\beta=1.70$ and $\beta=2.26$, respectively. These values are nearly equivalent to the horizontal and vertical cloud size scaling independent of cloud type and latitude, respectively, but differ from the horizontal scaling values calculated for the tropics and extratropical regions. 
The results of this investigation derive from data that form heavily skewed distributions. The following properties of these distributions pertain to the validity of our results. The mean is not an adequate statistic to summarize those PDFs because extreme values distort it. However, these extreme values do not distort the median since the median is based on ranks. When a power-law exponent is $\beta \leq 2$ (or $\beta \leq 3$ ), the mean (or the second moment) diverges. In this work, however, all lengths considered are finite and so are the moments. As a consequence, the horizontal cloud-chord-length mean and vertical cloud-chord-length standard deviation are dominated by the largest and most infrequent scales and are therefore unreliable. The median is thus preferable to interpret the data. Kiemle et al. (2015) also acknowledged this fact and provided the median cloud-gap lengths instead of their mean. With this limitation in mind, mean lengths were provided here to facilitate the comparison with published works where only the mean quantities were made available. Second, the prevalent fitting method used to calculate a power-law exponent (in general on a log-log plot) tends to provide biased estimates of the exponent (Goldstein et al. 2004); other alternative statistical techniques such as the maximum likelihood estimate used in this work (Newman 2005) or the Hill estimator (Resnick 2007) are also useful.

\section{Summary}

Horizontal and vertical cloud scales observed by CloudSat are quantified separately for each CloudSat cloud type and also for combinations of clouds independent of cloud type. Individual cloud types do not follow obvious power-law behavior as a function of horizontal or vertical scale. However, a robust power-law scaling of cloud horizontal length and vertical thickness is observed when all cloud types are combined. A fitting approach described in Wood and Field (2011) and the maximum likelihood estimation method of Newman (2005) are both used to calculate power-law exponents $\beta$. The horizontal-length exponent is approximated by $\beta \approx 1.66 \pm 0.00$ over two orders of magnitude $(\sim 10-1000 \mathrm{~km})$. The exponent of vertical thickness is approximated by $\beta \approx 2.23 \pm 0.00$ over one order of magnitude $(\sim 1-14 \mathrm{~km})$.

These exponents are in approximate agreement with previous studies using satellite, dropsonde, and in situ aircraft observations, and numerical model simulations. The anisotropy in the horizontal and vertical scaling is consistent with the scaling of horizontal wind in the horizontal dimension, while there is similarity to, but not a perfect match with, scaling of buoyancy variance in the vertical dimension. Careful estimation of $\beta$ with uncertainties is necessary to deduce the spatiotemporal behavior of atmospheric processes. The exponents reported here are not exactly analogous to those reported using aircraft data because of fundamental sampling differences of aircraft, which may fly inside, outside, and around clouds, and may avoid particular cloud types. Further investigations relating physical processes to the power laws in cloud-chord-length spectral exponents obtained with the vertically pointing $94-\mathrm{GHz}$ CloudSat radar are warranted.

Careful estimates of $\beta$ and its associated uncertainties are highly relevant to deducing the spatial behavior of atmospheric processes. Two independent estimates of $\beta$ obtained from different methodologies may lead to different conclusions if they are inconsistent. Typically, previous investigations either have made qualitative comparisons against the exponent benchmarks of $-5 / 3$ or -3 or have not considered uncertainties in the calculated exponents.

Certain theoretical arguments explain some aspects of our observations. The Kolmogorov-Obukhov theory predicts an energy spectrum exponent of $-5 / 3$ for homogeneous and isotropic turbulence. However, our results and others clearly show an anisotropy between the horizontal and vertical directions. The BolgianoObukhov theory predicts an energy spectrum exponent $-11 / 5$, but the range of scales for which it is valid is mutually exclusive with the Kolmogorov-Obukhov regime and would confine the Kolmogorov-Obukhov to scales much smaller than those observed (Lovejoy and Schertzer 2013). The 23/9-dimensional KolmogorovBolgiano-Obukhov does predict an anisotropy $\beta_{h} / \beta_{v}=$ $(-5 / 3) /(-11 / 5)$ between the horizontal and vertical dimensions similar to the results described herein. Yet, our observations concern cloud chord length whereas Kolmogorov-Bolgiano-Obukhov predicts spectral exponents. Further research is warranted on linking together cloud chord length in the horizontal and vertical with theoretical constructs of energy and buoyancy fluxes.

Numerical GCM simulations of weather and climate variability and change should produce scaling exponents that are consistent with present-day observations, including horizontal and vertical cloud-chord-length probability distributions. As noted by O'Brien et al. (2013), observations of scale dependence in vertical and horizontal cloud chord length are needed to advance understanding of resolution dependence in climate and weather prediction models, and of the subgrid schemes used to represent cloud processes. In climate models, convective clouds and the associated high-level stratiform clouds are determined by the triggering of cumulus parameterizations and the subsequent cloud microphysical schemes, while low-level stratiform clouds are determined by boundary layer schemes with the 
subsequent cloud microphysical schemes. Observed scaling behavior in the vertical dimension provides constraints to test if the different subgrid-scale parameterizations are triggered and interact with each other properly in different atmospheric conditions (e.g., tropical conditions with convective instability vs extratropical conditions with baroclinic instability). Our results obtained from CloudSat observations extend to subgrid scales and indicate robust anisotropy between the horizontal and vertical scaling of the cloud chord length. Our results should serve as a benchmark in evaluating the scale dependence of anisotropy within climate and weather numerical prediction models.

Acknowledgments. Part of this research was carried out at the Jet Propulsion Laboratory (JPL), California Institute of Technology, under a contract with the National Aeronautics and Space Administration, Grant NNH12ZDA001N. We thank two reviewers for insightful comments that led to a significantly improved manuscript. This project was supported by NASA's Making Earth Science Data Records for Use in Research Environments (MEaSUREs) program. CloudSat data were obtained through the CloudSat Data Processing Center (http://www.cloudsat.cira.colostate.edu/). Government sponsorship acknowledged.

\section{REFERENCES}

Bacmeister, J. T., and G. L. Stephens, 2011: Spatial statistics of likely convective clouds in CloudSat data. J. Geophys. Res., 116, D04104, https://doi.org/10.1029/2010JD014444.

Barker, H. W., Z. Qu, S. Bélair, S. Leroyer, J. A. Milbrandt, and P. A. Vaillancourt, 2017: Scaling properties of observed and simulated satellite visible radiances. J. Geophys. Res. Atmos., 122, 9413-9429, https://doi.org/10.1002/2017JD027146.

Bony, S., and Coauthors, 2015: Clouds, circulation and climate sensitivity. Nat. Geosci., 8, 261-268, https://doi.org/10.1038/ngeo2398.

Cahalan, R. F., W. Ridgway, W. J. Wiscombe, T. L. Bell, and J. B. Snider, 1994: The albedo of fractal stratocumulus clouds. J. Atmos. Sci., 51, 2434-2455, https://doi.org/10.1175/15200469(1994)051<2434:TAOFSC > 2.0.CO;2.

Cho, J. Y. N., and Coauthors, 1999: Horizontal wavenumber spectra of winds, temperature, and trace gases during the Pacific Exploratory Missions: 1. Climatology. J. Geophys. Res., 104, 5697-5716, https://doi.org/10.1029/98JD01825.

Clauset, A., C. R. Shalizi, and M. E. J. Newman, 2009: Power-law distributions in empirical data. SIAM Rev., 51, 661-703, https://doi.org/10.1137/070710111.

Cusack, S., J. M. Edwards, and R. Kershaw, 1999: Estimating the subgrid variance of saturation, and its parameterization for use in a GCM cloud scheme. Quart. J. Roy. Meteor. Soc., 125, 3057-3076, https://doi.org/10.1002/qj.49712556013.

Davis, A. B., and A. Marshak, 2010: Solar radiation transport in the cloudy atmosphere: A 3D perspective on observations and climate impacts. Rep. Prog. Phys., 73, 026801, https://doi.org/ 10.1088/0034-4885/73/2/026801.
Fischer, L., C. Kiemle, and G. C. Craig, 2012: Height-resolved variability of midlatitude tropospheric water vapor measured by an airborne lidar. Geophys. Res. Lett., 39, L06803, https:// doi.org/10.1029/2011GL050621.

_ G. C. Craig, and C. Kiemle, 2013: Horizontal structure function and vertical correlation analysis of mesoscale water vapor variability observed by airborne lidar. J. Geophys. Res. Atmos., 118, 7579-7590, https://doi.org/10.1002/jgrd.50588.

Goldstein, M. L., S. A. Morris, and G. G. Yen, 2004: Problems with fitting to the power-law distribution. Eur. Phys. J., 41B, 255 258, https://doi.org/10.1140/epjb/e2004-00316-5.

Hamilton, K., Y. O. Takahashi, and W. Ohfuchi, 2008: Mesoscale spectrum of atmospheric motions investigated in a very fine resolution global general circulation model. J. Geophys. Res., 113, D18110, https://doi.org/10.1029/2008JD009785.

Holloway, C. E., A. A. Wing, S. Bony, C. Muller, H. Masunaga, T. S. L'Ecuyer, D. D. Turner, and P. Zuidema, 2017: Observing convective aggregation. Surv. Geophys., 38, 11991236, https://doi.org/10.1007/s10712-017-9419-1.

IPCC, 2013: Climate Change 2013: The Physical Science Basis. Cambridge University Press, 1535 pp., https://doi.org/10.1017/ CBO9781107415324.

Kahn, B. H., and J. Teixeira, 2009: A global climatology of temperature and water vapor variance scaling from the atmospheric infrared sounder. J. Climate, 22, 5558-5576, https:// doi.org/10.1175/2009JCLI2934.1.

_ scaling in global models: Comparisons to satellite and aircraft data. J. Atmos. Sci., 68, 2156-2168, https://doi.org/10.1175/ 2011JAS3737.1.

Kiemle, C., G. Ehret, S. R. Kawa, and E. V. Browell, 2015: The global distribution of cloud gaps in CALIPSO data. J. Quant. Spectrosc. Radiat. Transfer, 153, 95-101, https://doi.org/ 10.1016/j.jqsrt.2014.12.001.

Landau, L. D., and E. M. Lifshitz, 1987: Fluid Mechanics. 2nd ed. Landau and Lifshitz: Course of Theoretical Physics, Vol. 6, Pergamon, $134 \mathrm{pp}$.

Lilley, M., S. Lovejoy, K. Strawbridge, and D. Schertzer, 2004: 23/9 dimensional anisotropic scaling of passive admixtures using lidar data of aerosols. Phys. Rev., 70E, 036307, https://doi.org/ 10.1103/PhysRevE.70.036307.

Lindborg, E., 2007: Horizontal wavenumber spectra of vertical vorticity and horizontal divergence in the upper troposphere and lower stratosphere. J. Atmos. Sci., 64, 1017-1025, https:// doi.org/10.1175/JAS3864.1.

Lovejoy, S., 1982: Area-perimeter for rain and cloud areas. Science, 216, 185-187, https://doi.org/10.1126/science.216.4542.185.

— Laws and Multifractal Cascades. Cambridge University Press, $505 \mathrm{pp}$.

— A. F. Tuck, S. J. Hovde, and D. Schertzer, 2007: Is isotropic turbulence relevant in the atmosphere? Geophys. Res. Lett., 34, L15802, https://doi.org/10.1029/2007GL029359.

—_, - _ , and D. Schertzer, 2010: Horizontal cascade structure of atmospheric fields determined from aircraft data. J. Geophys. Res., 115, D13105, https://doi.org/10.1029/ 2009JD013353.

Mauritsen, T., and B. Stevens, 2015: Missing iris effect as a possible cause of muted hydrological change and high climate sensitivity in models. Nat. Geosci., 8, 346-351, https://doi.org/ 10.1038/ngeo2414.

Miller, S. D., and Coauthors, 2014: Estimating three-dimensional cloud structure via statistically blended satellite observations. 
J. Appl. Meteor. Climatol., 53, 437-455, https://doi.org/ 10.1175/JAMC-D-13-070.1.

Nastrom, G. D., and K. S. Gage, 1985: A climatology of atmospheric wavenumber spectra of wind and temperature observed by commercial aircraft. J. Atmos. Sci., 42, 950-960, https://doi.org/ 10.1175/1520-0469(1985)042<0950:ACOAWS>2.0.CO;2.

— W. W. Jasperson, and K. S. Gage, 1986: Horizontal spectra of atmospheric tracers measured during the global atmospheric sampling program. J. Geophys. Res., 91, 13 201-13 209, https:// doi.org/10.1029/JD091iD12p13201.

Newman, M. E. J., 2005: Power laws, Pareto distributions and Zipf's law. Contemp. Phys., 46, 323-351, https://doi.org/ 10.1080/00107510500052444.

Noda, A. T., M. Satoh, Y. Yamada, C. Kodama, and T. Seiki, 2014: Responses of tropical and subtropical high-cloud statistics to global warming. J. Climate, 27, 7753-7768, https://doi.org/ 10.1175/JCLI-D-14-00179.1.

O'Brien, T. A., F. Li, W. D. Collins, S. A. Rauscher, T. D. Ringler, M. Taylor, S. M. Hagos, and L. R. Leung, 2013: Observed scaling in clouds and precipitation and scale incognizance in regional to global atmospheric models. J. Climate, 26, 9313-9333, https://doi.org/10.1175/JCLI-D-13-00005.1.

Pierrehumbert, R. T., 1996: Anomalous scaling of high cloud variability in the tropical Pacific. Geophys. Res. Lett., 23, 1095-1098, https://doi.org/10.1029/96GL01121.

Pressel, K. G., and W. D. Collins, 2012: First-order structure function analysis of statistical scale invariance in the AIRSobserved water vapor field. J. Climate, 25, 5538-5555, https:// doi.org/10.1175/JCLI-D-11-00374.1.

_ _ _ and A. R. Desai, 2014: The spatial scale dependence of water vapor variability inferred from observations from a very tall tower. J. Geophys. Res. Atmos., 119, 9822-9837, https:// doi.org/10.1002/2013JD021141.

Resnick, S. I., 2007: Heavy-Tail Phenomena: Probabilistic and Statistical Modeling. Springer, $404 \mathrm{pp}$.

Sassen, K., and Z. Wang, 2005: Level 2 cloud scenario classification product process description and interface control document. Cooperative Institute for Research in the Atmosphere, Colorado State University, Fort Collins, CO, 36 pp., http://cswww.cira.colostate.edu/ICD/2B-CLDCLASS/ 2B-CLDCLASS_PDICD_4.0.pdf.

- , and - 2008: Classifying clouds around the globe with the CloudSat radar: 1-year of results. Geophys. Res. Lett., 35, L04805, https://doi.org/10.1029/2007GL032591.

Schemann, V., B. Stevens, V. Grützun, and J. Quaas, 2013: Scale dependency of total water variance and its implication for cloud parameterizations. J. Atmos. Sci., 70, 3615-3630, https:// doi.org/10.1175/JAS-D-13-09.1.
Schertzer, D., and S. Lovejoy, 1983: Elliptical turbulence in the atmosphere. Proc. Fourth Symp. on Turbulent Shear Flows, Karlsruhle, West Germany, 11.1-11.8.

- , and -1985 : Generalised scale invariance in turbulent phenomena. PhysicoChem. Hydrodyn., 6, 623-635.

Siebesma, A. P., and H. J. J. Jonker, 2000: Anomalous scaling of cumulus cloud boundaries. Phys. Rev. Lett., 85, 214-217, https://doi.org/10.1103/PhysRevLett.85.214.

Skamarock, W. C., 2004: Evaluating mesoscale NWP models using kinetic energy spectra. Mon. Wea. Rev., 132, 3019-3032, https://doi.org/10.1175/MWR2830.1.

Stephens, G. L., and Coauthors, 2002: The CloudSat mission and the A-Train: A new dimension of space-based observations of clouds and precipitation. Bull. Amer. Meteor. Soc., 83, 17711790, https://doi.org/10.1175/BAMS-83-12-1771.

Takahashi, Y. O., K. Hamilton, and W. Ohfuchi, 2006: Explicit global simulation of the mesoscale spectrum of atmospheric motions. Geophys. Res. Lett., 33, L12812, https://doi.org/ 10.1029/2006GL026429.

Tjemkes, S. A., and M. Visser, 1994: Horizontal variability of temperature, specific humidity, and cloud liquid water as derived from spaceborne observations. J. Geophys. Res., 99, 23 089-23 105, https://doi.org/10.1029/94JD01718.

Tuck, A. F., 2010: From molecules to meteorology via turbulent scale invariance. Quart. J. Roy. Meteor. Soc., 136, 1125-1144, https://doi.org/10.1002/qj.644; Corrigendum, 137, 275, https:// doi.org/10.1002/qj.780.

Wing, A. A., and K. A. Emanuel, 2014: Physical mechanisms controlling self-aggregation of convection in idealized numerical modeling simulations. J. Adv. Model. Earth Syst., 6, 59-74, https://doi.org/10.1002/2013MS000269.

Wood, R., and J. P. Taylor, 2001: Liquid water path variability in unbroken marine stratocumulus cloud. Quart. J. Roy. Meteor. Soc., 127, 2635-2662, https://doi.org/10.1002/qj.49712757807.

— , and P. R. Field, 2011: The distribution of cloud horizontal sizes. J. Climate, 24, 4800-4816, https://doi.org/10.1175/ 2011JCLI4056.1.

Zelinka, M. D., C. Zhou, and S. A. Klein, 2016: Insights from a refined decomposition of cloud feedbacks. Geophys. Res. Lett., 43, 9259-9269, https://doi.org/10.1002/2016GL069917.

Zhang, D., T. Luo, D. Liu, and Z. Wang, 2014: Spatial scales of altocumulus clouds observed with collocated CALIPSO and CloudSat measurements. Atmos. Res., 149, 58-69, https:// doi.org/10.1016/j.atmosres.2014.05.023.

Zhao, G., and L. Di Girolamo, 2007: Statistics on the macrophysical properties of trade wind cumuli over the tropical western Atlantic. J. Geophys. Res., 112, D10204, https:// doi.org/10.1029/2006JD007371. 